\title{
Loss of SPARC-mediated VEGFR-1 suppression after injury reveals a novel antiangiogenic activity of VEGF-A
}

Miho Nozaki, ${ }^{1}$ Eiji Sakurai,,1,2 Brian J. Raisler,,1,3 Judit Z. Baffi, ${ }^{1}$ Jassir Witta, ${ }^{4}$ Yuichiro Ogura, ${ }^{2}$ Rolf A. Brekken, ${ }^{5}$ E. Helene Sage, ${ }^{6}$ Balamurali K. Ambati, ${ }^{7}$ and Jayakrishna Ambati ${ }^{1,3}$

${ }^{1}$ Department of Ophthalmology and Visual Sciences, University of Kentucky, Lexington, Kentucky, USA. ${ }^{2}$ Department of Ophthalmology, Nagoya City University Medical School, Nagoya, Japan. ${ }^{3}$ Department of Physiology and 4Department of Internal Medicine, University of Kentucky, Lexington, Kentucky, USA. ${ }^{5}$ Department of Surgery, University of Texas Southwestern Medical Center, Dallas, Texas, USA. ${ }^{6}$ Hope Heart Program, Benaroya Research Institute at Virginia Mason, Seattle, Washington, USA. ${ }^{7}$ Department of Ophthalmology, Medical College of Georgia, Augusta, Georgia, USA.

VEGF-A promotes angiogenesis in many tissues. Here we report that choroidal neovascularization (CNV) incited by injury was increased by excess VEGF-A before injury but was suppressed by VEGF-A after injury. This unorthodox antiangiogenic effect was mediated via VEGFR-1 activation and VEGFR-2 deactivation, the latter via Src homology domain 2-containing (SH2-containing) tyrosine phosphatase-1 (SHP-1). The VEGFR-1-specific ligand placental growth factor-1 (PIGF-1), but not VEGF-E, which selectively binds VEGFR-2, mimicked these responses. Excess VEGF-A increased CNV before injury because VEGFR-1 activation was silenced by secreted protein, acidic and rich in cysteine (SPARC). The transient decline of SPARC after injury revealed a temporal window in which VEGF-A signaling was routed principally through VEGFR-1. These observations indicate that therapeutic design of VEGF-A inhibition should include consideration of the level and activity of SPARC.

\section{Introduction}

VEGF-A, which signals through the receptor tyrosine kinases VEGFR-1 and VEGFR-2, plays a dominant role in physiologic and pathologic angiogenesis, with VEGFR-2 implicated as its principal proangiogenic transducer. The function of VEGFR-1 is more nebulous. In vitro studies in porcine aortic (1) or human umbilical vein endothelial cells $(2,3)$ demonstrate that VEGFR-1 repressed VEGFR-2-mediated cell proliferation through active signaling. Deletion of Vegfr 1 in mice results in embryonic lethality due to endothelial overcrowding and disorganized vasculature (4). However, vascular development is grossly unaffected in mice with a deletion of the VEGFR-1 kinase domain (Vegfr1 tk-/-) (5), which suggests that VEGFR-1 subserves a negative role in embryogenesis by acting as a trap that modulates endogenous VEGF-A levels. In pathologic angiogenesis, however, conflicting data about VEGFR-1 function have emerged from studies demonstrating that it both amplifies $(6,7)$ and antagonizes (8) neovascularization. VEGFR-1 signaling therefore appears to be both cell/tissue specific and context/stage dependent.

Choroidal neovascularization (CNV) is the principal cause of blindness in patients with age-related macular degeneration (AMD), a condition responsible for vision loss in 25-30 million people worldwide in total (9). VEGF-A is present in CNV membranes surgically excised from patients with AMD (10), and pharmacological inhibition of VEGF-A decreases experimental laser-

Nonstandard abbreviations used: ALK1, activin receptor-like kinase 1; AMD, age-related macular degeneration; BMOV, bis(maltolato)oxovanadium(IV); CNV, choroidal neovascularization; PAI-1, plasminogen activator inhibitor type I; PlGF-1, placental growth factor-1; PTP, protein tyrosine phosphatase; RPE, retinal pigmented epithelium; SH2, Src homology domain 2; SHP-1, SH2-containing tyrosine phosphatase-1; SPARC, secreted protein, acidic and rich in cysteine.

Conflict of interest: J. Ambati is listed on an initial patent filing by the University of Kentucky describing these findings.

Citation for this article: J. Clin. Invest. 116:422-429 (2006). doi:10.1172/JCI26316. induced CNV (11). These data are the bases for current clinical trials of anti-VEGF-A therapy in patients with AMD.

However, the precise role of VEGF-A in CNV remains unclear. Whereas subretinal injection of viral vectors encoding VEGF-A leads to retinal pigmented epithelium (RPE) overexpression of VEGF-A and subsequent CNV (12), increased production of VEGF-A directed by RPE-specific promoters, such as RPE65 or VMD2, does not produce CNV $(13,14)$. However, enhancement of VEGF-A coupled with subretinal injection of null viral vector induced $\mathrm{CNV}(14)$, suggesting that increased VEGF-A alone is insufficient to induce CNV without concomitant mechanical trauma or immune deviation. A clinical trial of an anti-VEGF-A aptamer (pegaptanib) in CNV demonstrated an inverse dose response in visual outcome (15). Further, although the lowest dose decreased the rate of vision loss, it did not prevent an increase in CNV lesion size.

It is now appreciated that activities of VEGF-A and other cytokines are influenced by the interaction of cells with the ECM. Matricellular proteins regulate cell-ECM communication and thereby can influence many remodeling events, including angiogenesis (16). We focused on the matricellular protein SPARC (secreted protein, acidic and rich in cysteine) because it has been reported to decrease the stimulatory activity of VEGF-A on endothelial cell proliferation, in part by its abrogation of VEGFR-1 phosphorylation (17).

\section{Results}

VEGF-A inbibits CNV via VEGFR-1. We tested the effect of injecting VEGF-A into the vitreous cavity of mice after laser injury. This injury fractures Bruch membrane, the ECM between the RPE and choroid, the highly vascular tissue beneath the RPE, and triggers proliferation of choroidal endothelial cells that subsequently migrate through these fractures, resulting in $\operatorname{CNV}(18,19)$. Intravitreous injection of VEGF-A (0.02-0.95 pmol), either immediately following or 1 day after laser injury, decreased the volume of $\mathrm{CNV}$ in a dose-dependent fashion at both 1 (Figure 1, A and B) 


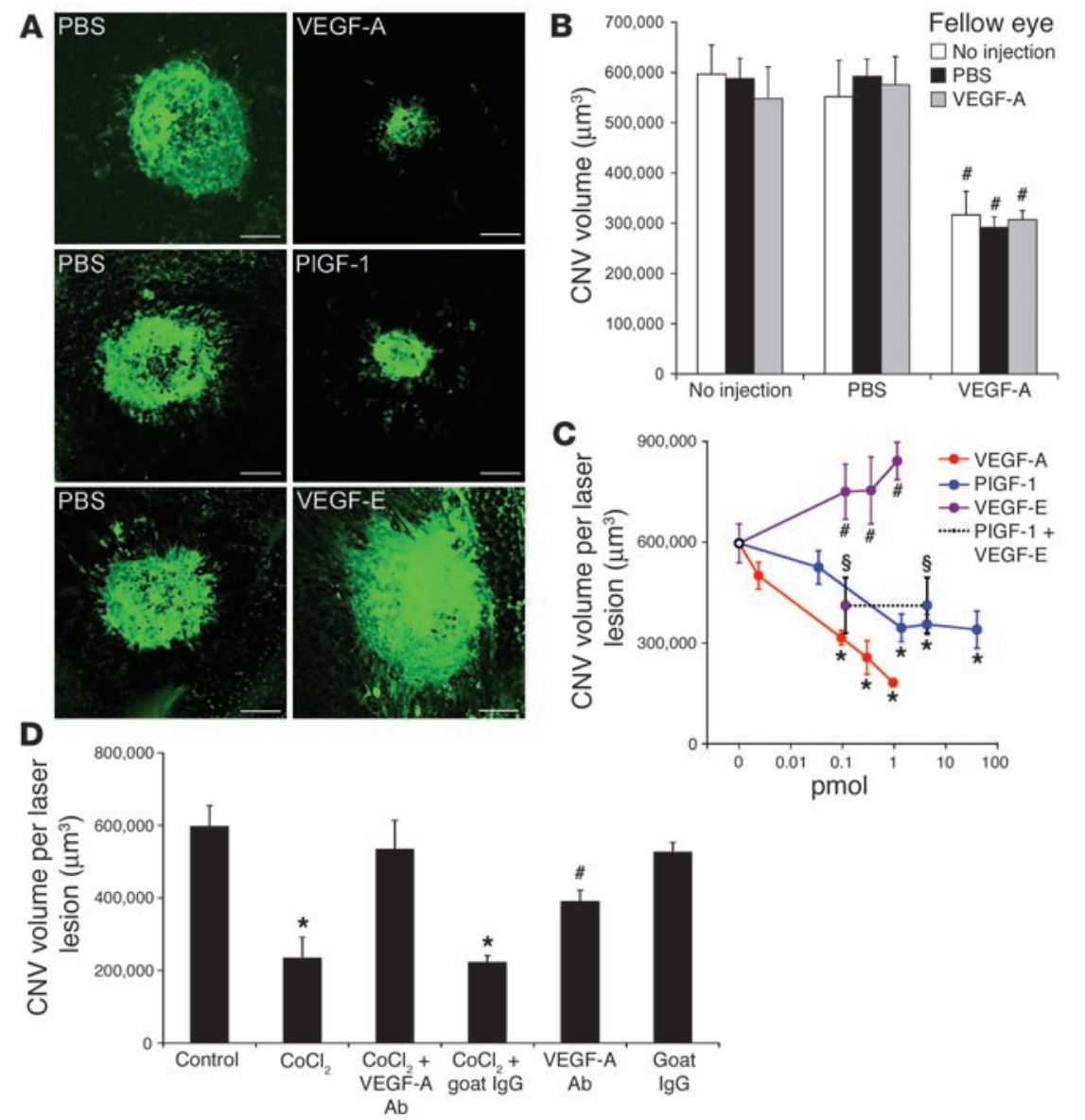

Figure 1

VEGFR-1 ligands suppressed CNV. (A) Stacked confocal image of representative laser-induced CNV lesions in PBS-treated eyes were larger than in VEGF-A-treated (0.29 pmol) or PIGF-1treated $(4.3 \mathrm{pmol})$ eyes and smaller than in VEGF-E-treated (0.34 pmol) eyes. Scale bars: $100 \mu \mathrm{m}$. (B) PBS treatment alone did not reduce CNV, and VEGF-A $(0.1 \mathrm{pmol})$ reduced CNV regardless of the treatment of the fellow eye. $n=15-46$ per data point. $\# P<0.05$ compared with corresponding uninjected or PBS-treated groups. (C) VEGF-A (red; $n=38-46$ per data point) and PIGF-1 (blue; $n=18$ per data point) reduced CNV at 1 week after injury in a dosedependent fashion. VEGF-E (purple) and PIGF-1 together (single data point depicted as dotted line) suppressed CNV similar to PIGF-1 alone. $n=12$ per data point. VEGF-E increased CNV. $n=12 .{ }^{*} P<0.01,{ }^{\#} P<0.05$ compared with PBS; $\$ P<0.01$ compared with VEGF-E; $P>0.90$ compared with PIGF-1. (D) CNV inhibited by $\mathrm{CoCl}_{2}(0.77 \mathrm{nmol})$ was abrogated by anti-VEGF-A antibody ( $6.7 \mathrm{fmol}$ ) but not by control goat IgG (6.7 fmol). Anti-VEGF-A antibody modestly reduced $\mathrm{CNV}$ compared with goat IgG. ${ }^{*} P<0.01$ compared with control (PBS). $\sharp P<0.05$ compared with goat IgG. $n=18-24$ per data point. and 2 weeks (data not shown) after laser injury. CNV volume in eyes injected with vehicle (PBS) alone did not differ significantly from that in the uninjected fellow eyes; further, CNV volume in eyes treated with VEGF-A did not depend on whether the fellow eye was uninjected or treated with PBS or VEGF-A (Figure 1C).

To test whether endogenous VEGF-A would induce similar effects, we injected $\mathrm{CoCl}_{2}$, which increases VEGF-A expression. Intravitreous injection of $\mathrm{CoCl}_{2}(0.77 \mathrm{nmol}) 1$ day after laser injury decreased the volume of CNV compared with PBS or no injection; this inhibition was abrogated by neutralizing anti-VEGF-A antibody but not by isotype control IgG (Figure 1D). We confirmed the functional specificity of this anti-VEGF-A antibody by demonstrating that it modestly inhibited CNV, in comparison with control IgG, consistent with previous reports of VEGF-A blockade (11). Although $\mathrm{CoCl}_{2}$ can induce pleiotropic effects in cells, reversal of the antiangiogenic effect by specific neutralization of VEGF-A confirms its involvement in suppressing CNV. Because hypoxia has been speculated to be involved in CNV (reviewed in ref. 20), the effects of $\mathrm{CoCl}_{2}$ might be pathophysiologically relevant.

Because VEGF-A binds both VEGFR-1 and VEGFR-2, we tested the effects of placental growth factor-1 (PlGF-1), a VEGFR-1-specific ligand (21), and VEGF-E, a VEGFR-2-specific ligand (22). Intravitreous injection of PlGF-1 (0.03-43.1 pmol) resulted in similar dose-dependent suppressive effects on CNV, whereas VEGF-E (0.11-1.14 pmol) did not (Figure 1B). Inhibition of CNV by VEGF-A or $\mathrm{CoCl}_{2}$ was abolished by neutralizing antibody against VEGFR-1 (40 pmol; $\left.\mathrm{IC}_{50}=13.3-53.3 \mathrm{nM}\right)$ but not by anti-VEGFR-2 (1.7 pmol; $\left.\mathrm{IC}_{50}=0.7-2 \mathrm{nM}\right)$ or by isotype control antibody $(40 \mathrm{pmol}$ ) (Figure
2A). At the doses used, intravitreous injection of anti-VEGFR-2 antibody modestly reduced CNV, confirming a prior report (23); however, intravitreous injection of anti-VEGFR-1 antibody did not reduce $\mathrm{CNV}$, a result different from a prior report of systemic VEGFR-1 blockade (24) (discussed below). The modest increase in CNV induced by VEGF-E was blocked by anti-VEGFR-2 antibody but not anti-VEGFR-1 or isotype control antibody, confirming the specificity and effectiveness of these doses (Figure 2A). That CNV reduction induced by $\mathrm{PlGF}-1$ was not enhanced by coadministration of VEGF-E suggests that cooperation between VEGFR-1 and VEGFR-2 is not required for antiangiogenic activity (Figure 1B). Although VEGF-E alone increased CNV volume, it was unable to overcome the antiangiogenic effect of PlGF-1, demonstrating the dominance of VEGFR-1 over VEGFR-2 in this context.

VEGFR-1 blockade augmented the increase in CNV induced by VEGF-E (Figure 2A); in the setting of exogenously triggered selective VEGFR-2 signaling, endogenous VEGFR-1 activation therefore appears to function as a negative regulator of angiogenesis. Administration of VEGF-A and $\mathrm{CoCl}_{2}$ increased CNV in Vegfr-1 $t k^{-/}$mice, results opposite of those seen in wild-type mice and supportive of a negative regulatory function for VEGFR-1 during this angiogenic response (Figure $2 \mathrm{~B}$ ). Collectively, these data demonstrate that the suppressive effects of VEGF-A occur through active VEGFR-1 signaling and not by its functioning as a decoy receptor sequestering ligand from VEGFR-2.

Endogenous VEGF-A induces a zone of angiogenic inbibition. These data may be relevant to clinical observations. Specifically, ophthalmologists have noted that there is often a single area of CNV in 

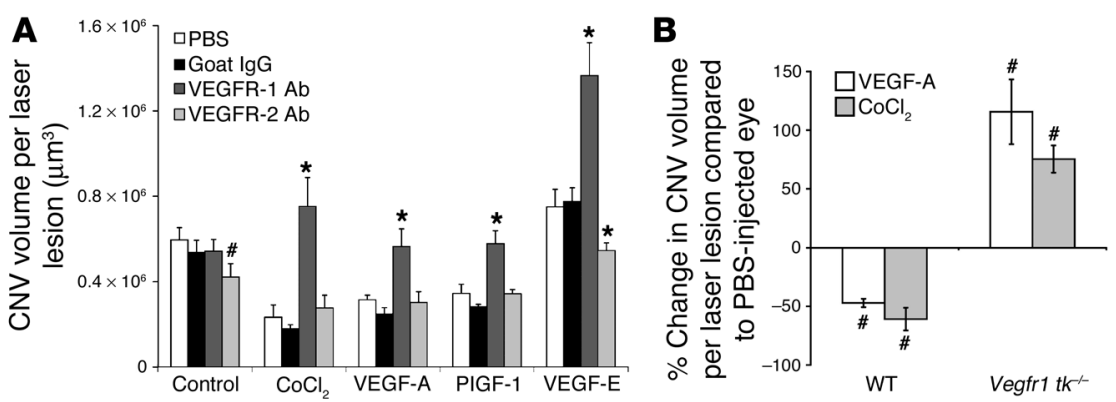

Figure 2

VEGF-A reduced CNV via VEGFR-1. (A) Anti-VEGFR-1 Ab (40 pmol), but not anti-VEGFR-2 $\mathrm{Ab}(1.7 \mathrm{pmol})$, abrogated inhibition of CNV by $\mathrm{CoCl}_{2}$, VEGF-A, and PIGF-1. ${ }^{*} P<0.01 \mathrm{com}-$ pared with drug alone. Antibody against VEGFR-2 but not VEGFR-1 modestly reduced CNV compared with goat IgG (40 pmol). ${ }^{\#} P<0.05$ compared with goat IgG. $n=10-12$ per data point. (B) VEGF-A and $\mathrm{CoCl}_{2}$ reduced CNV in wild-type mice but increased it in Vegfr1 tk $\mathrm{k}^{-1-}$ mice compared with PBS-injected fellow eyes. ${ }^{\#} P<0.05$ compared with PBS. $n=10$ per data point. $\mathrm{CoCl}_{2}, 0.77$ nmol; VEGF-A, 0.1 pmol; VEGF-E, 0.11 pmol.

patients with AMD despite widespread disease, that is, thickening of the inner aspect of Bruch's membrane throughout the RPE and choroid (N.M. Bressler and W.F. Mieler, personal communication). Even when multiple foci exist, typically the ingrowth channels of $\mathrm{CNV}$ through Bruch membrane are separated by 1 to $2 \mathrm{~mm}$, with several intervening fractures in Bruch membrane not containing CNV (H.E. Grossniklaus and G.A. Lutty, personal communication). Interestingly, therapeutic destruction of CNV nearly always results in recurrence in the immediate vicinity. We speculated that these phenomena result from high levels of VEGF-A emanating from the existing focus of $\mathrm{CNV}$ and that these excessive amounts create a "zone of inhibition" that suppresses formation of adjacent $\mathrm{CNV}$.

We tested to determine whether a similar zone of inhibition exists in the mouse by

\section{Figure 3}

Preexisting CNV inhibited subsequent CNV via VEGF-A. (A and B) Representative choroidal flat mounts show that CNV lesions (insets show magnified images) were smaller in eyes (A) where preexisting laser injury (area denoted by arrow) was performed 2 days earlier compared with those in eyes (B) without prior injury. Arrowheads denote optic nerve. Scale bars: $200 \mu \mathrm{m}$. (C) Preexisting laser injury created 2 days before subsequent injury markedly decreased CNV in the subsequent laser spots near $(\sim 0.75 \mathrm{~mm})$ the preexisting injury and slightly decreased CNV in spots far $(\sim 1.25$ $\mathrm{mm}$ ) from it. Preexisting laser injury created 14 days before subsequent injury in wildtype mice did not affect CNV of subsequent laser spots either near or far from preexisting injury. ${ }^{*} P<0.01$ compared with eyes without preexisting injury; ${ }^{\#} P<0.05$ compared with far lesions in the day 2 group. $n=12$ per data point. (D) Neutralizing anti-VEGF-A antibody reversed this inhibition in a dose-dependent manner. ${ }^{\#} P<0.05$ compared with no injection. $n=12$ per data point. (C and $\mathbf{D})$.
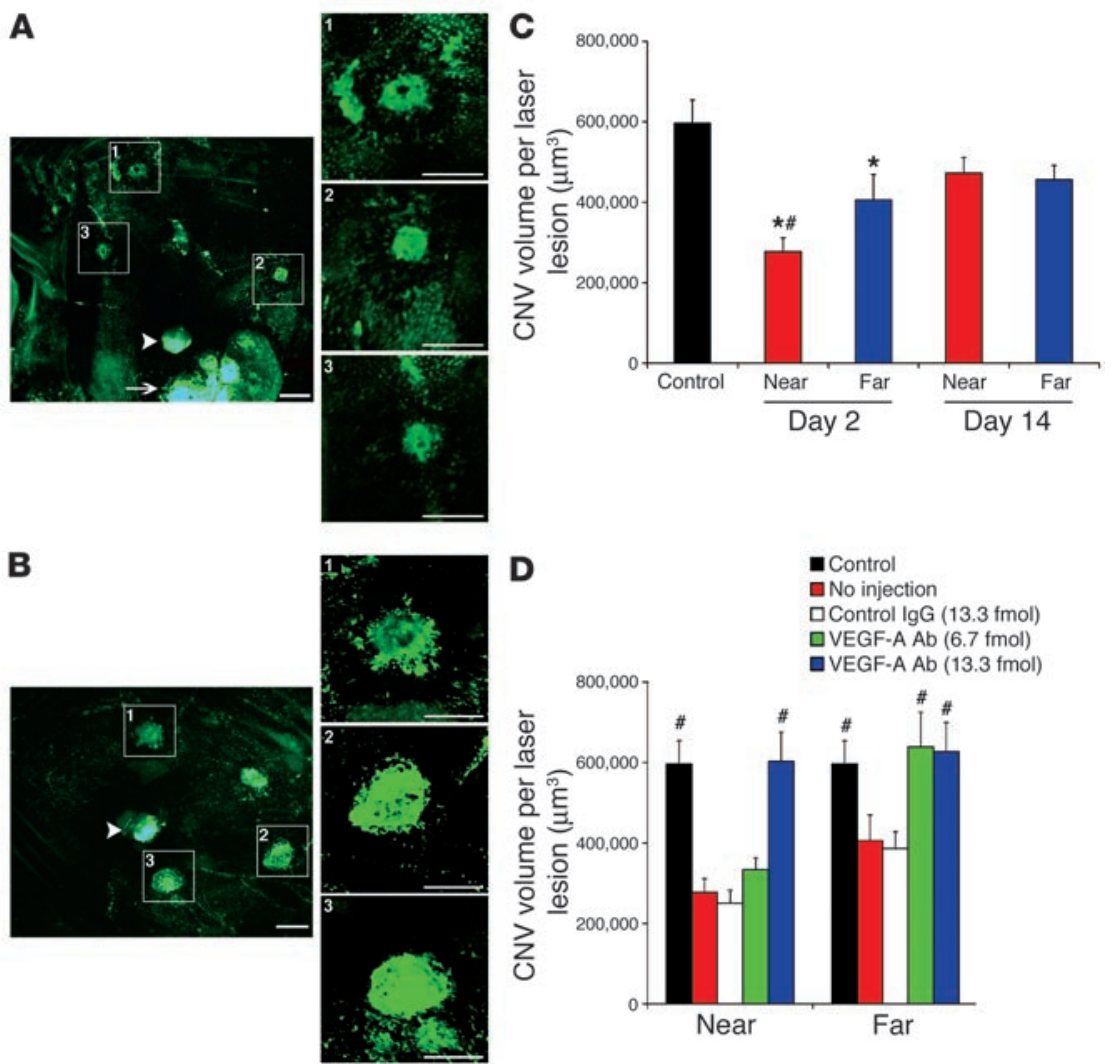

placement of laser spots at 2 different distances $(\sim 0.75 \mathrm{~mm}$ and $\sim 1.25 \mathrm{~mm})$ from an area of preexisting laser injury performed 2 days earlier. The initial injury was placed 2 days earlier because the peak of its VEGF-A response, which occurs 3 days after injury (25), would occur on the day after the secondary injury, a time point when exogenous VEGF-A injection leads to CNV suppression (Figure 1B). $\mathrm{CNV}$ volume was significantly decreased in the subsequent laser spots, more so in those nearer to $(53.5 \% \pm 5.7 \% ; P<0.01)$ than farther from $(31.9 \% \pm 10.7 \% ; P=0.05)$ the preexisting injury sites (Figure 3, A-C). Neutralizing anti-VEGF-A antibody, but not control goat $\mathrm{IgG}$, injected on the day interv ening between the initial and subsequent laser injuries reversed inhibition of $\mathrm{CNV}$; a lower dose (6.7 fmol) was required to restore normal CNV volume in the distant laser spots and a higher dose $(13.3 \mathrm{fmol})$ for the spots closer to the preexisting injury (Figure 3D). This is consistent with the notion that diffusion of VEGF-A from the original injury suppresses CNV in subsequent lesions. When laser spots were placed 2 weeks after initial injury, there was no significant CNV inhibition $(P>0.34)$ (Figure 3C), consistent with absence of excess VEGF-A at this time point (25). These data not only provide a mechanistic basis for the clinical observations but also provide insight into why anti-VEGF-A therapies do not maintain the short-term success seen in patients with $\mathrm{CNV}$ and why they exhibit an inverse dose-response curve (15). 
A
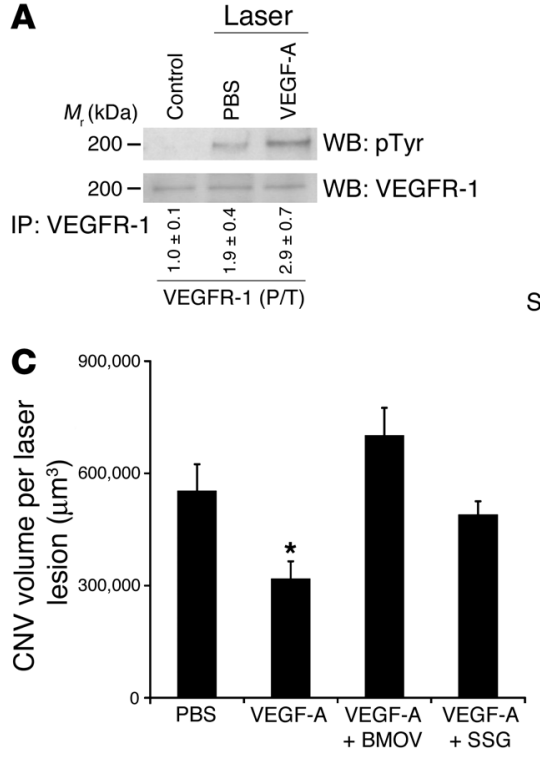

B

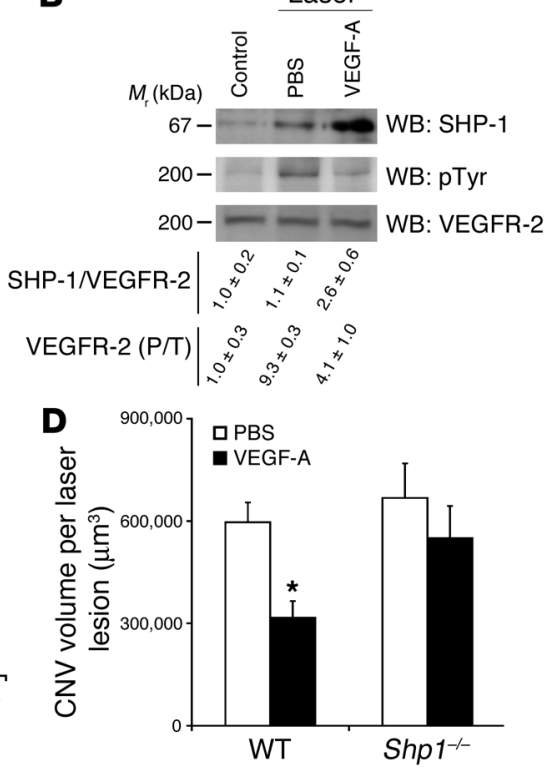

\section{Figure 4}

VEGF-A decreased CNV through VEGFR-1-induced negative transduction of VEGFR-2 via SHP-1. (A) Representative figure shows VEGFR-1 phosphorylation levels in RPE/choroid of eyes before injury (control) and 30 minutes after intravitreous injection of PBS or VEGF-A, 1 day after injury. $n=5$. WB, Western blot. (B) Representative figure shows that VEGF-A, injected 1 day after laser injury, increased interaction of SHP-1 with VEGFR-2 and reduced VEGFR-2 phosphorylation at 30 minutes after injection, 1 day after injury, and before injury (control), without affecting VEGFR-2 expression. Densitometric ratios of SHP-1 to total VEGFR-2 and of phosphorylated (P) to total (T) VEGFR-1 or VEGFR-2 are shown before (control) and after injury. Ratios were normalized to control values. $n=5$. (C) VEGF-A-induced CNV suppression was abrogated by BMOV $(0.16 \mu \mathrm{mol})$ and sodium stibogluconate (SSG) $(0.56 \mathrm{nmol})$ in wild-type mice. $n=12$ per data point. (D) VEGF-A did not significantly suppress CNV in Shp $1^{-1-}$ mice $(n=12$ per data point; $P=0.44)$. ${ }^{*} P<0.05$ compared with PBS. VEGF-A, 0.1 pmol.
VEGF-A ligation of VEGFR-1 negatively regulates VEGFR-2 signaling via SHP-1. We studied whether VEGF-A executes its antiangiogenic program by directly promoting VEGFR-1 activity or antagonizing VEGFR-2 activity. VEGFR-1 tyrosine kinase phosphorylation was markedly enhanced following injection of VEGF-A 1 day after laser injury (Figure 4A). Simultaneously, we observed increased interaction between the protein tyrosine phosphatase (PTP) Src homology domain 2-containing (SH2-containing) tyrosine phosphatase-1 (SHP-1) and the VEGFR-2 complex as well as a reduction in VEGFR-2 phosphorylation (Figure 4B). These findings are compatible with a model in which VEGFR-1 negatively regulates VEGFR-2 activation via SHP-1, although conclusive evidence of a direct effect awaits development of a VEGFR-1-specific tyrosine kinase inhibitor. We confirmed that VEGFR-2 dephosphorylation translated into a functional inhibition of angiogenesis because the pan-PTP inhibitor bis(maltolato) oxovanadium(IV) (BMOV) abrogated VEGF-A-induced inhibition of $\mathrm{CNV}$ (Figure 4C). Sodium stibogluconate, a potent SHP-1 inhibitor (26), also reversed VEGF-A-induced inhibition of CNV (Figure 4C). Further, we demonstrated that $S h p 1^{-/-}$mice were resistant to VEGF-Ainduced CNV suppression (Figure 4D). Collectively, these data confirm a specific effect of VEGF-A on SHP-1. We also observed that VEGF-A did not modulate the interaction between VEGFR-2 and other PTPs, such as PTP1B and human low molecular weight cytoplasmic PTP (HCPTPA) (data not shown). These data demonstrate that, after laser injury, excess VEGF-A negatively regulates VEGFR-2 signaling and identify a potentially novel mechanism by which VEGF-A can modulate its own angiogenic action.

$S P A R C$ regulates $V E G F-A$-induced effects on $C N V$. In contrast to their antiangiogenic action when injected after laser injury, VEGF-A and $\mathrm{CoCl}_{2}$ increased $\mathrm{CNV}$ when injected 1 day before injury (Figure 5). This was mediated via VEGFR-2 signaling because VEGF-E, but not PlGF-1, promoted CNV when injected 1 day before injury (Figure 5). In most tissues, VEGFR-1 autophosphorylation was weak, possibly because it is constitutively repressed. Therefore, we postulated the presence of a protein that would restrain VEGFR-1 kinase at rest and would itself be diminished after injury, thereby relieving the functional block of VEGFR-1 activation.
One such candidate is SPARC, which we previously demonstrated binds to VEGF-A and inhibits the kinase activity of VEGFR-1 but not VEGFR-2 in vitro (17). SPARC was constitutively expressed in the RPE/choroid, but its expression declined after injury and recovered to near-baseline levels 2 days later (Figure 6A). We also identified a specific binding interaction between SPARC and VEGF-A in the mouse eye in vivo (Figure 6B); this interaction was specific because VEGF-A was not bound to IGF-binding protein-3 (IGFBP-3), a matricellular protein like SPARC (Figure 6C). Suppression of CNV induced by VEGF-A injected 1 day after injury, when levels of SPARC were declining, was dose dependently abolished by recombinant human SPARC (2.5-7.5 pmol), which is $92 \%$ identical to mouse SPARC (Figure 6D) (27). Restoration of CNV by exogenous SPARC was essentially eliminated by a neutralizing antibody against SPARC, confirming the specificity of this response (Figure 6D). The anti-SPARC IgG (26.7-80 pmol) inhibited the augmented CNV resulting from VEGF-A injected 1 day before injury, when SPARC levels were high (Figure 6E).

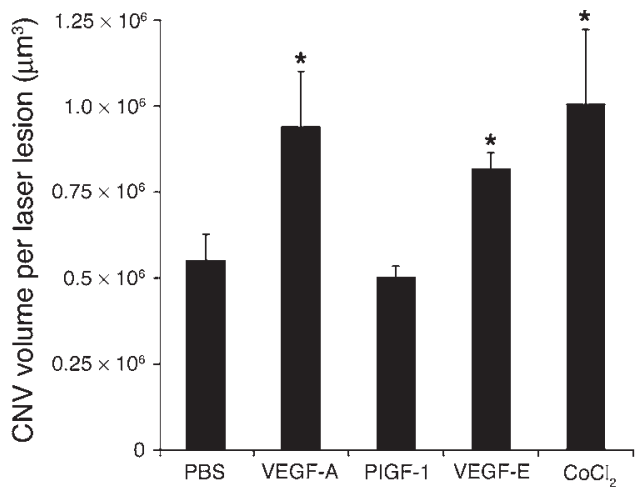

Figure 5

VEGF-A increased CNV before injury via VEGFR-2. Injected 1 day before injury, VEGF-A (0.1 pmol), VEGF-E (0.11 pmol), and $\mathrm{CoCl}_{2}$ (0.77 nmol), but not PIGF-1 (43.1 pmol), increased CNV. ${ }^{*} P<0.05$ compared with PBS. $n=10$ per data point. 

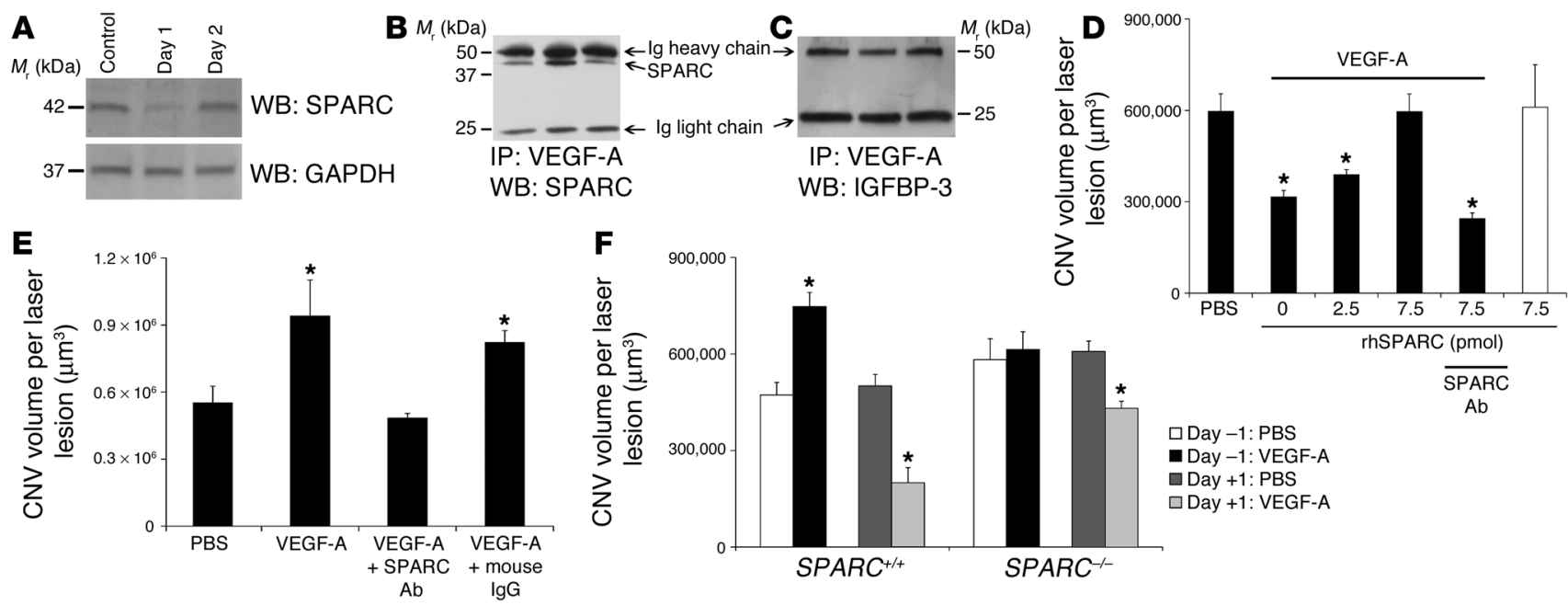

\section{Figure 6}

SPARC regulated VEGF-A effects on CNV. (A) Constitutive (control) RPE/choroid expression of SPARC was decreased 1 day after laser injury and recovered 2 days after injury. Figure is representative of 3 experiments. (B and $\mathbf{C}$ ) Immunoprecipitation of 3 independent samples revealed that VEGF-A interacted with SPARC (B) but not IGF binding protein-3 (IGFBP-3) (C) in vivo. (D) CNV inhibition by VEGF-A injected 1 day after injury was abolished by recombinant human SPARC (rhSPARC) and restored by neutralizing anti-SPARC Ab (80 pmol). ${ }^{*} P<0.05 \mathrm{compared}$ with PBS. Recombinant SPARC injection alone (white bar) did not change CNV compared with PBS. (E) Neutralizing anti-SPARC Ab (26.7 pmol) but not control mouse IgG abrogated CNV increase induced by VEGF-A 1 day before injury. ${ }^{*} P<0.05$ compared with PBS. (F) VEGF-A injected 1 day before laser injury increased CNV in SPARC $C^{+/+}$mice but not in SPARC ${ }^{-/-}$mice. VEGF-A injected 1 day after laser injury decreased CNV in $S P A R C^{+/+}$and $S P A R C^{-/-}$mice. ${ }^{*} P<0.05$ compared with PBS injection on the same day. VEGF-A, 0.1 pmol. $n=10-14$ per data point (D-F).

The pro- and antiangiogenic actions of VEGF-A injected before versus after injury, respectively, were duplicated in $\mathrm{Sparc}^{+/+}$mice. However, in Sparc ${ }^{-1}$ mice, the proangiogenic response of VEGF-A injected 1 day before injury was eliminated, whereas the antiangiogenic response of VEGF-A injected 1 day after injury was preserved (Figure 6F). Of note, the basal CNV response in $\mathrm{Sparc}^{-1-}$ eyes was modestly but significantly greater than in $\mathrm{Sparc}^{+/+}$eyes, consistent with the diminished ECM characteristic of Sparc-/- mice that has been proposed as permissive for enhanced tumor growth (28). The modest difference between Sparc ${ }^{+/+}$mice and C57BL/6J mice in basal CNV response is attributed to the genetic variation arising from their mixed background. Collectively, these data indicate that SPARC limits the capacity of excess VEGF-A to suppress CNV via VEGFR-1 and that the transient decline of SPARC in the wake of injury unmasks the antiangiogenic action of VEGF-A due to unsilencing of VEGFR-1 activation.

\section{Discussion}

Historically, VEGFR-1 was assigned a role as a nonsignaling decoy receptor because of the low activity and embryonic dispensability of its tyrosine kinase function. More recently, its role has become more interesting because VEGFR-1 signaling has been reported both to promote $(6,7)$ and suppress $(8)$ VEGF-A-driven angiogenesis. We report not only that PlGF-1 inhibits inflammatory CNV, extending the scope of VEGFR-1 function, but also what we believe is the unprecedented observation that VEGF-A itself can suppress angiogenesis. Multiple lines of evidence emerging from genetic ablation, antibody neutralization, and receptor-selective ligand activation all strongly support the thesis that the antiangiogenic action of VEGF-A is mediated by VEGFR-1. Previously, VEGF-A has been reported to reduce VEGF-E-induced VEGFR-2 tyrosine kinase phosphorylation in capillary endothelial cells in vitro, raising the provocative hypothesis that VEGF-A could limit its own angiogenic activity through VEGFR-1 (29). We have presented in vivo confirmation of this hypothesis. These findings contribute significantly to our understanding of the consequences of the interaction of VEGFR-1 with its ligands and describe a unique mechanism by which VEGFR-1 regulates angiogenesis.

Prior reports have noted that the biological effects of VEGF-A are dose dependent. These observations are consistent with our data, which indicate that high levels of VEGF-A cannot sustain, and indeed may inhibit, CNV. Exogenous VEGF-A in the myocardium or skeletal muscle can lead to dysregulated vasculogenesis $(30,31)$ and disrupt embryonic intersomitic artery formation (32). In addition, exogenous VEGF-A can inhibit smooth muscle cell proliferation, without affecting angiogenesis $(33,34)$. However, we believe our data are the first to demonstrate direct arrest of endothelial cell proliferation and frank inhibition of angiogenesis by VEGF-A.

These data do not contradict the well-described angiogenic properties of VEGF-A. Indeed, our data indicating that intravitreous injection of antibodies against VEGF-A or VEGFR-2 reduces laserinduced CNV confirm findings of other investigators using different antagonists $(11,23)$. Rather, they unveil an unrecognized ability of excess VEGF-A in the postinjury setting to suppress angiogenesis by predominantly activating VEGFR-1 signaling and preventing endothelial cells from responding to mitogenic signals. It appears that the system is exquisitely sensitive to the precise level of VEGF-A in the immediate postinjury period and responds differently if that level exceeds the concentration of VEGF-A induced by injury. The "switch" that diverts the injured tissue away from proliferation is driven through VEGFR-1 signaling, which appears to be dominant in an environment of excess VEGF-A, in contrast to the usual dominance of VEGFR-2. Unraveling this antagonistic pathway of excess VEGF-A and comparing it with those of existing angiogenic inhibitors may reveal additional robust and powerful therapeutic targets in the panoply of angiogenesis-driven disorders. 
Rakic and colleagues demonstrated that systemic administration of anti-VEGFR-1 antibody inhibits experimental CNV (24), while we found that intravitreous delivery did not. This divergence could arise from the fact that cell populations are affected differentially by local versus systemic VEGFR-1 blockade. Systemic but not intravitreous VEGFR-1 blockade would interfere with mobilization of bone marrow-derived progenitor cells (35) that are known to contribute to $\operatorname{CNV}(36,37)$. This difference is clinically relevant as most antiVEGF-A therapies for CNV rely on intravitreous delivery to minimize potential adverse effects of systemic VEGF-A antagonism.

Rakic et al. (24) also demonstrated that laser-induced CNV is inhibited in $\mathrm{Plg}^{-1-}$ mice, which is consistent with the role of PlGF in the bone marrow, where it mobilizes progenitors either directly by recruiting VEGFR-1+ cells or indirectly by inducing matrix metalloproteinase-9, which increases progenitor cell proliferation and motility via release of soluble Kit ligand (35). In contrast, intravitreous injection of PlGF, which inhibits CNV when administered after injury, would not execute such effects on the bone marrow. These differences also might have emerged because exogenous and endogenous PlGF may heterodimerize with VEGF-A differently in $\mathrm{CNV}$. The role of these heterodimers is controversial because they have been reported both to promote (38) and antagonize (39) neovascularization. An analogous variation is observed in experimental retinal neovascularization, which is inhibited both by genetic ablation of $P \lg f(6)$ and by intravitreous administration of PlGF (40), although the latter may be due to increased cell survival. A similar divergence in angiogenic response to endogenous and exogenous proteins exists in the case of other cytokines, such as plasminogen activator inhibitor type I (PAI-1). Indeed, Rakic and colleagues also have shown that laser-induced CNV is inhibited both in Pai-/- mice and in PAI-1-treated wild-type mice (41). The apparently different effects of endogenous and exogenous PlGF also might reflect modulation of plasminogen activator activity by VEGFR-1 activation $(42,43)$.

Reduction in CNV in $\mathrm{Plg}^{-/-}$mice also is intriguing because these mice express other VEGFR-1 ligands, including VEGF-A (6). This raises the formal possibility that reduced $\mathrm{CNV}$ in $\mathrm{Plg} f^{-/}$mice might reflect molecular or developmental plasticity in response to gene disruption rather than PlGF deficiency alone. Secondary developmental effects induced by the altered vascular phenotype account for a similar divergence phenomenon in $A l k 1^{-/-}$mice, which display enhanced angiogenesis (44), while enforced activin receptor-like kinase 1 (ALK1) expression stimulates endothelial cell migration and proliferation (45). It also is possible that subtle defects in ocular vascular development in $P l g f^{-/-}$mice reported by Carmeliet and colleagues (6) might have influenced their $\mathrm{CNV}$ responses.

These data add VEGF-A to the group of cytokines, such as angiopoietin-1 (46-48), nitric oxide $(49,50)$, pigment epitheliumderived factor $(51-53)$, and TGF- $\beta(45,54)$, whose modulation of angiogenesis displays context-dependent bidirectionality. The paradoxical effect of VEGF-A resembles that of TGF- $\beta$, which is proangiogenic at low doses and antiangiogenic at high doses, an effect attributed to differential activation of the TGF- $\beta$ receptors ALK1 and ALK5 (45), akin to the differential routing via VEGFR-1 and VEGFR-2. This Janus-like effect reveals novel therapeutic strategies to modulate angiogenesis in the setting of inflammation and highlights the importance of developing assays for markers such as SPARC to target therapeutics more specifically.

In most systems, VEGFR-1 tyrosine kinase activity has been described as weak. Our findings provide a paradigm by which its activation is controlled in vivo by SPARC and illustrate the multifunctional nature of this receptor in promoting or curtailing angiogenesis. These data might also explain the divergent outcomes of experiments involving VEGFR-1 function in different angiogenesis models. The poor intrinsic kinase activity of VEGFR-1 in many systems may be due to repressive elements in its juxtamembrane domain (55). It is also possible that VEGFR-1 signaling could occur without phosphorylation of consensus-positive regulatory tyrosine residues, i.e., VEGFR-1 could be a poor substrate for itself. Further investigation of the role of SPARC in maintaining repression of VEGFR-1 may improve our fragmentary understanding of its activation, particularly during development and in cancer, where this receptor appears functional. In addition, the propensity of the macula to develop CNV despite widespread subretinal disease in AMD may be related to the concentration of SPARC in this central region in monkeys (56) (and presumably in humans), which may prevent downregulation of proangiogenic VEGFR-2 signaling by VEGFR-1.

The expression and activity of SPARC are segregated largely to tissues that are undergoing remodeling or turnover. As such, it is well placed to regulate the activity of potent growth factors such as VEGF-A, which often are the primary stimuli for remodeling of the local microenvironment. The present study provides a physiological example of how SPARC potentially regulates the angiogenic process. The initiation of angiogenesis by soluble factors such as VEGF-A is complex and involves the activation of individual endothelial cells that express both VEGFR-1 and VEGFR-2. Our data point to extracellular adaptor proteins as critical linchpins in the induction of the VEGFR-2-driven angiogenic program. Previous studies (17) showed that SPARC can interfere with VEGF-A ligation of VEGFR-1 and that SPARC-mediated inhibition of VEGF-A-induced phosphorylation of VEGFR-1 was long lived (at least 24 hours). In the context of $\mathrm{CNV}$, this time frame is relevant to the demonstrated activity of VEGF-A in that setting. Investigations using mice with a deficiency in a matricellular protein (e.g., thrombospondin-1 and/or -2, the SPARC ortholog hevin, or SPARC) support the hypothesis that the function of matricellular proteins is contextual and that regulated expression of these proteins is important for maintenance of tissue homeostasis and responses to injury $(16,57,58)$.

The basal CNV response of $S p a r c^{-/-}$mice was greater than that of wild-type counterparts (Figure 6D), a result consistent with the heightened neovascular response in sponge implants reported in Sparc ${ }^{-/-}$animals as well as the synergistic increase in vascularization in the foreign body response of SPARC/hevin double-null mice $(59,60)$. The inhibitory effect of SPARC on vessel growth resides in part in the $\mathrm{C}$ terminal, $\mathrm{Ca}^{+2}$-binding $\mathrm{EF}$ hand, whereas other peptides released by plasmin or stromelysin-1 stimulate angiogenesis by affecting the cell cycle and/or migration of endothelial cells (27, 61). Our present study emphasizes the contextual dependence of SPARC and its activity as a regulator of angiogenesis.

Our data define a previously unrecognized autoregulatory potential of excess VEGF-A and are relevant to ongoing clinical trials examining anti-VEGF-A therapy in AMD and VEGF-A over-expression in ischemic limb and cardiac disease. Data from the pegaptanib clinical trials in patients with AMD showed an inverse therapeutic dose response (15), indicating that partial inhibition of VEGF-A could be optimal. The inability of this drug to arrest the increasing size of the $\mathrm{CNV}$ lesion with time in patients with AMD indicates that VEGF-A might have dual actions in the human eye as well. However, that 
pegaptanib decreased the rate of vascular leakage points to a divergent response of VEGF-A in mediating growth versus hyperpermeability of new blood vessels. Whether similar bifurcation of VEGF-A signaling occurs in laser-induced CNV warrants investigation.

Our findings provide insight into the context and stage dependency of the role of VEGF-A in ocular neovascularization as well as interactions between a matricellular protein and a VEGF receptor that regulate and route signaling by VEGF-A. Our findings also demonstrate alternative pathways of VEGF-A signal transduction and emphasize the need to clarify the complex effects of interactions among VEGF-A, its receptors, SPARC, and the chemokine network in ocular neovascularization before VEGF-A alone can be considered an ideal therapeutic target.

\section{Methods}

Animals. Wild-type C57BL/6 mice were purchased from Jackson Laboratory. Vegf $r$ - 1 th $\mathrm{t}^{-/-}$mice were generated as described previously (5) and backcrossed 6-10 times to C57BL/6 animals. Sparc ${ }^{-/-}$and $\mathrm{Sparc}^{+/+}$mice on a mixed C57BL/6 $\times 129 / \mathrm{SvJ}$ background have been described (62). Mice (6- to 8-week-old males) were anesthetized by intraperitoneal injection of ketamine (50 mg/kg; Fort Dodge Animal Health) and xylazine $(10 \mathrm{mg} / \mathrm{kg}$; Phoenix Scientific Inc.), and pupils were dilated with topical tropicamide (1\%; Alcon). Experiments were approved by the University of Kentucky Institutional Animal Care and Use Committee.

CNV. Laser photocoagulation $(532 \mathrm{~nm}, 200 \mathrm{~mW}, 100 \mathrm{~ms}, 75 \mu \mathrm{m})$ (OcuLight GL; IRIDEX Corp.) was performed on both eyes (3 spots per eye) of each animal to induce $\mathrm{CNV}$ as described $(25,63)$. $\mathrm{CNV}$ volumes were measured by scanning laser confocal microscope (TCS SP; Leica) as reported $(25,63)$, with $0.5 \%$ FITC-conjugated Griffonia simplicifolia isolectin B4 (Vector Laboratories) or 0.5\% FITC-conjugated rat anti-mouse CD31 (BD Biosciences), or by cardiac perfusion with $5 \mathrm{mg} / \mathrm{ml}$ FITC-dextran $\left(2 \times 10^{6}\right.$ average weight; Sigma-Aldrich). Pairwise comparisons among volumes obtained by lectin, CD31, and dextran staining were highly correlated $\left(r^{2}>0.90\right)$. CNV volumes per laser lesion were compared by hierarchical logistic regression using repeated measures analysis as described $(25,63)$. Results are expressed as mean $\pm \operatorname{SEM}$ ( $n$ refers to number of animals). Type I error not exceeding 0.05 was deemed significant.

Drug treatments. VEGF-A 164 (0.02-0.95 pmol), PlGF-1 (0.03-43.1 pmol), neutralizing goat antibodies against (a) mouse VEGF-A (6.7-13.3 fmol), (b) mouse VEGFR-1 (6.7-40 pmol), (c) mouse VEGFR-2 (0.17-1.7 pmol; all R\&D Systems); $\mathrm{CoCl}_{2}(0.77 \mathrm{nmol}$; Sigma-Aldrich), recombinant human SPARC (64) (2.5-7.5 pmol), neutralizing mouse antibody against human SPARC (65) (clone 293; 26.7-80 pmol; cross-reactive with mouse SPARC), VEGF-E (0.11-1.14 pmol; Cell Sciences), BMOV (0.16 $\mu \mathrm{mol}$; gift of J.H. McNeill and C. Orvig, University of British Columbia, Vancouver, Canada), or sodium stibogluconate (SSG) $(0.56 \mathrm{nmol})$ were dissolved in PBS (SigmaAldrich), and injected into the vitreous cavity in a total volume of $1 \mu \mathrm{l}$ with a 33-gauge Exmire microsyringe (Ito Corp.).
Western blotting. Equal amounts of total protein from RPE/choroid were resolved on SDS 4-20\% polyacrylamide gradient gels and transferred to nitrocellulose membranes for Western blotting with goat antibody against mouse SPARC (1:500; R\&D Systems). Loading was assessed by blotting with rabbit antibody against human GAPDH (1:2,000; Abcam).

Immunoprecipitation and immunoblotting. RPE/choroid lysates were immunoprecipitated with goat antibodies against mouse VEGFR-1 or VEGFR-2 ( $8 \mu \mathrm{g} / \mathrm{ml}$; R\&D Systems) that were immobilized on protein G-agarose, subjected to SDS-PAGE, immunoblotted with mouse monoclonal antibody against phosphotyrosine (1:1,000; Upstate USA Inc.), and subsequently reprobed with rabbit antibodies against human VEGFR-1 or mouse VEGFR-2 (1:500; Santa Cruz Biotechnology Inc.). SPARC-VEGF-A interaction was identified by immunoprecipitation with mouse antibody against human VEGF-A (66) (clone 12D7; $4 \mu \mathrm{g} / \mathrm{ml}$; cross-reactive with mouse VEGF-A) and immunoblotting with mouse antibody against human SPARC (clone 293; $0.75 \mu \mathrm{g} / \mathrm{ml}$ ) or with goat antibody against mouse IGFBP-3 $(0.1 \mu \mathrm{g} / \mathrm{ml}$; R\&D Systems)

\section{Acknowledgments}

We thank M. Shibuya for the generous gift of breeder Vegfr-1 tt ${ }^{-/-}$mice; S. Funk, M.J. Hanson, S. Joshi, R. King, N. Mezei, and G. Workman for technical assistance; and R. Mohan, S. Bondada, M.W. Fannon, V. Kumar, P.A. Pearson, A.M. Rao, and G.S. Rao for illuminating discussions. J. Ambati is supported by NIH EY-15422 and the Dennis W. Jahnigen Career Development Award, administered by the American Geriatrics Society, and funded by the John A. Hartford Foundation and Atlantic Philanthropies, the Macula Vision Research Foundation, the International Retinal Research Foundation, the E. Matilda Ziegler Foundation for the Blind, a physician-scientist award from the University of Kentucky, and a departmental challenge grant from Research to Prevent Blindness; M. Nozaki by ARVO/Japan National Society for the Prevention of Blindness; E. Sakurai by Fight For Sight; J.Z. Baffi and B.J. Raisler by NIH DC-000065; B.K. Ambati by a VA Career Development Award and the Knights Templar Eye Foundation; and E.H. Sage by NIH GM-40711 and EY-13180.

Received for publication July 18, 2005, and accepted in revised form November 8, 2005.

Miho Nozaki, Eiji Sakurai, Brian J. Raisler, Judit Z. Baffi, and Jassir Witta contributed equally to this work.

Address correspondence to: Jayakrishna Ambati, Department of Ophthalmology and Visual Sciences, University of Kentucky, 740 S. Limestone Street, Lexington, Kentucky 40536-0284, USA. Phone: (859) 323-5867, ext. 259; Fax: (859) 323-1122; E-mail: jamba2@uky.edu.
1. Rahimi, N., Dayanir, V., and Lashkari, K. 2000 Receptor chimeras indicate that the vascular endothelial growth factor receptor-1 (VEGFR-1) modulates mitogenic activity of VEGFR-2 in endothelial cells. J. Biol. Chem. 275:16986-16992.

2.Zeng, H., Dvorak, H.F., and Mukhopadhyay, D 2001. Vascular permeability factor (VPF)/vascular endothelial growth factor (VEGF) receptor-1 down-modulates VPF/VEGF receptor-2-mediated endothelial cell proliferation, but not migration, through phosphatidylinositol 3-kinase-dependent pathways. J. Biol. Chem. 276:26969-26979.

3. Kontos, C.D., Mason, J., and Ahmed, A. 2001. Vascular endothelial growth factor receptor-1 modulates vascular endothelial growth factor-mediated angiogenesis via nitric oxide. Am. J. Pathol. 159:993-1008.

4. Fong, G., Zhang, L., Bryce, D., and Peng, J. 1999. Increased hemangioblast commitment, not vascular disorganization, is the primary defect in flt- 1 knock-out mice. Development. 126:3015-3025.

5. Hiratsuka, S., Minowa, O., Kuno, J., Noda, T., and Shibuya, M. 1998. Flt-1 lacking the tyrosine kinase domain is sufficient for normal development and angiogenesis in mice. Proc. Natl. Acad. Sci. U. S. A. 95:9349-9354.

6. Carmeliet, P., et al. 2001. Synergism between vascular endothelial growth factor and placental growth factor contributes to angiogenesis and plasma extravasation in pathological conditions. Nat. Med. 7:575-583.

7. Hiratsuka, S., et al. 2001. Involvement of flt-1 tyrosine kinase (vascular endothelial growth factor receptor-1) in pathological angiogenesis. Cancer Res. 61:1207-1213.

8. Bussolati, B., et al. 2001. Vascular endothelial growth factor receptor-1 modulates vascular endothelial growth factor-mediated angiogenesis via nitric oxide. Am. J. Pathol. 159:993-1008.

9. Smith, W., et al. 2001. Risk factors for age-related macular degeneration: pooled findings from three continents. Ophthalmology. 108:697-704.

10. Lopez, P.F., et al. 1996. Transdifferentiated retinal 
pigment epithelial cells are immunoreactive for vascular endothelial growth factor in surgically excised age-related macular degeneration-related choroidal neovascular membranes. Invest. Ophthalmol. Vis. Sci. 37:855-868.

11. Saishin, Y., et al. 2003. VEGF-TRAP R1R2 $_{\text {suppresses }}$ choroidal neovascularization and VEGF-induced breakdown of the blood-retinal barrier. J. Cell. Physiol. 195:241-248.

12. Spilsbury, K., et al. 2000. Overexpression of vascular endothelial growth factor (VEGF) in the retinal pigment epithelium leads to the development of choroidal neovascularization. Am. J. Pathol. 157:135-144.

13. Schwesinger, C., et al. 2001. Intrachoroidal neovascularization in transgenic mice overexpressing vascular endothelial growth factor in the retinal pigment epithelium. Am. J. Pathol. 158:1161-1172.

14. Oshima, Y., et al. 2004. Increased expression of VEGF in retinal pigmented epithelial cells is not sufficient to cause choroidal neovascularization. J. Cell. Physiol. 201:393-400.

15. Gragoudas, E.S., et al. 2004. Pegaptanib for neovascular age-related macular degeneration. N. Engl. J. Med. 351:2805-2816.

16. Bornstein, P., and Sage, E.H. 2002. Matricellular proteins: extracellular modulators of cell function. Curr. Opin. Cell Biol. 14:608-616.

17. Kupprion, C., Motamed, K., and Sage, E.H. 1998. SPARC (BM-40, osteonectin) inhibits the mitogenic effect of vascular endothelial growth factor on microvascular endothelial cells. J. Biol. Chem. 273:29635-29640.

18. Ryan, S.J. 1982. Subretinal neovascularization. Natural history of an experimental model. Arch. Ophthalmol. 100:1804-1809.

19. Tobe, T., et al. 1998. Targeted disruption of the FGF2 gene does not prevent choroidal neovascularization in a murine model. Am. J. Pathol. 153:1641-1646.

20. Ambati, J., et al. 2003. Age-related macular degeneration: etiology, pathogenesis, and therapeutic strategies. Surv. Ophthalmol. 48:257-293.

21. Park, J., et al. 1994. Placenta growth factor. Potentiation of vascular endothelial growth factor bioactivity, in vitro and in vivo, and high affinity binding to Flt-1 but not to Flk-1/KDR. J. Biol. Chem. 269:25646-25654.

22. Ogawa, S., et al. 1998. A novel type of vascular endothelial growth factor, VEGF-E (NZ-7 VEGF), preferentially utilizes KDR/Flk-1 receptor and carries a potent mitotic activity without heparin-binding domain. J. Biol. Chem. 273:31273-31282.

23. Takeda, A., et al. 2003. Suppression of experimental choroidal neovascularization utilizing KDR selective receptor tyrosine kinase inhibitor. Graefes Arch. Clin. Exp. Ophthalmol. 241:765-772.

24. Rakic, J.-M., et al. 2003. Placental growth factor, a member of the VEGF family, contributes to the development of choroidal neovascularization. Invest. Ophthalmol. Vis. Sci. 44:3186-3193.

25. Sakurai, E., et al. 2003. Macrophage depletion inhibits experimental choroidal neovascularization. Invest. Ophthalmol. Vis. Sci. 44:3578-3585.

26. Pathak, M.K., and Yi, T. 2001. Sodium stibogluconate is a potent inhibitor of protein tyrosine phosphatases and augments cytokine responses in hemopoietic cell lines. J. Immunol. 167:3391-3397.

27. Brekken, R.A., and Sage, E.H. 2001. SPARC, a matricellular protein: at the crossroads of cellmatrix communication. Matrix Biol. 19:816-827.

28. Brekken, R.A., et al. 2003. Enhanced growth of tumors in SPARC null mice is associated with changes in the ECM. J. Clin. Invest. 111:487-495. doi:10.1172/JCI200316804.

29. Autiero, M., et al. 2003. Role of PlGF in the intra- and intermolecular cross talk between the VEGF receptors Flt1 and Flk1. Nat. Med. 9:936-943.

30. Springer, M.L., et al. 1998. VEGF gene delivery to muscle: potential role for vasculogenesis in adults. Mol. Cell. 2:549-558.

31. Lee, R.J., et al. 2000. VEGF gene delivery to myocardium: deleterious effects of unregulated expression. Circulation. 102:898-901.

32. Drake, C., and Little, C. 1995. Exogenous vascular endothelial growth factor induces malformed and hyperfused vessels during embryonic neovascularization. Proc. Natl. Acad. Sci. U. S. A. 92:7657-7661.

33. Dorafshar, A.H., et al. 2003. Vascular endothelial growth factor inhibits mitogen-induced vascular smooth muscle cell proliferation. J. Surg. Res. 114:179-186

34. Laitinen, M., et al. 1997. VEGF gene transfer reduces intimal thickening via increased production of nitric oxide in carotid arteries. Hum. Gene Ther. 8:1737-1744.

35. Hattori, K., et al. 2002. Placental growth factor reconstitutes hematopoiesis by recruiting VEGFR1(+) stem cells from bone-marrow microenvironment. Nat. Med. 8:841-849.

36. Espinosa-Heidmann, D.G., Caicedo, A., Hernandez, E.P., Csaky, K.G., and Cousins, S.W. 2003. Bone marrow-derived progenitor cells contribute to experimental choroidal neovascularization. Invest. Ophthalmol. Vis. Sci. 44:4914-4919.

37. Sengupta, N., et al. 2003. The role of adult bone marrow-derived stem cells in choroidal neovascularization. Invest. Ophthalmol. Vis. Sci. 44:4908-4913.

38. Cao, Y., Linden, P., Shima, D., Browne, F., and Folkman, J. 1996. In vivo angiogenic activity and hypoxia induction of heterodimers of placenta growth factor/vascular endothelial growth factor. J. Clin. Invest. 98:2507-2511.

39. Eriksson, A., et al. 2002. Placenta growth factor-1 antagonizes VEGF-induced angiogenesis and tumor growth by the formation of functionally inactive PIGF-1/VEGF heterodimers. Cancer Cell. 1:99-108.

40. Shih, S.-C., Ju, M., Liu, N., and Smith, L.E.H. 2003. Selective stimulation of VEGFR-1 prevents oxygen-induced retinal vascular degeneration in retinopathy of prematurity. J. Clin. Invest. 112:50-57. doi:10.1172/JCI200317808

41. Lambert, V., et al. 2003. Dose-dependent modulation of choroidal neovascularization by plasminogen activator inhibitor type I: implications for clinical trials. Invest. Ophthalmol. Vis. Sci. 44:2791-2797.

42. Olofsson, B., et al. 1998. Vascular endothelial growth factor B (VEGF-B) binds to VEGF receptor-1 and regulates plasminogen activator activity in endothelial cells. Proc. Natl. Acad. Sci. U. S. A. 95:11709-11714.

43. Landgren, E., Schiller, P., Cao, Y., and ClaessonWelsh, L. 1998. Placenta growth factor stimulates MAP kinase and mitogenicity but not phospholipase C-gamma and migration of endothelial cells expressing Flt 1. Oncogene. 16:359-367.

44. Oh, S.P., et al. 2000. Activin receptor-like kinase 1 modulates transforming growth factor-beta 1 signaling in the regulation of angiogenesis. Proc. Natl. Acad. Sci. U. S. A. 97:2626-2631.

45. Goumans, M.-J., et al. 2002. Balancing the activation state of the endothelium via two distinct TGF- $\beta$ type I receptors. EMBO J. 21:1743-1753.

46. Suri, C., et al. 1998. Increased vascularization in mice overexpressing angiopoietin-1. Science. 282:468-471.

47. Visconti, R.P., Richardson, C.D., and Sato, T.N. 2002. Orchestration of angiogenesis and arteriovenous contribution by angiopoietins and vascular endothelial growth factor (VEGF). Proc. Natl. Acad.
Sci. U. S. A. 99:8219-8224.

48. Nambu, H., et al. 2004. Angiopoietin 1 inhibits ocular neovascularization and breakdown of the blood-retinal barrier. Gene Ther. 11:865-873.

49. Jadeski, L.C., and Lala, P.K. 1999. Nitric oxide synthase inhibition by $\mathrm{N}^{\mathrm{G}}$-nitro-L-arginine methyl ester inhibits tumor-induced angiogenesis in mammary tumors. Am. J. Pathol. 155:1381-1390.

50. Sennlaub, F., Courtois, Y., and Goureau, O. 1999. Nitric oxide synthase-II is expressed in severe corneal alkali burns and inhibits neovascularization. Invest. Ophthalmol. Vis. Sci. 40:2773-2779.

51. Mori, K., et al. 2001. Pigment epithelium-derived factor inhibits retinal and choroidal neovascularization. J. Cell. Physiol. 188:253-263.

52. Mori, K., et al. 2002. AAV-mediated gene transfer of pigment epithelium-derived factor inhibits choroidal neovascularization. Invest. Ophthalmol. Vis. Sci. 43:1994-2000.

53. Apte, R.S., Barreiro, R.A., Duh, E., Volpert, O., and Ferguson, T.A. 2004. Stimulation of neovascularization by the anti-angiogenic factor PEDF. Invest. Ophthalmol. Vis. Sci. 45:4491-4497.

54. Battegay, E.J., et al. 1990 . TGF- $\beta$ induces bimodal proliferation of connective tissue cells via complex control of an autocrine PDGF loop. Cell. 63:515-524.

55. Gille, H., et al. 2000. A repressor sequence in the juxtamembrane domain of Flt-1 (VEGFR-1) constitutively inhibits vascular endothelial growth factor-dependent phosphatidylinositol 3'-kinase activation and endothelial cell migration. EMBO J. 19:4064-4073.

56. Rodriguez, I.R., Moreira, E.F., Bok, D., and Kantorow, M. 2000. Osteonectin/SPARC secreted by $\mathrm{RPE}$ and localized to the outer plexiform layer of the monkey retina. Invest. Ophthalmol. Vis. Sci. 41:2438-2444.

57. Framson, P.E., and Sage, E.H. 2004. SPARC and tumor growth: where the seed meets the soil? J. Cell. Biochem. 92:679-690.

58. Sullivan, M.M., and Sage, E.H. 2004. Hevin/SC1, a matricellular glycoprotein and potential tumorsuppressor of the SPARC/BM-40/osteonectin family. Int. J. Biochem. Cell Biol. 36:991-996.

59. Bradshaw, A.D., et al. 2001. Increased fibrovascular invasion of subcutaneous polyvinyl alcohol sponges in SPARC-null mice. Wound Repair Regen. 9:522-530.

60. Barker, T.H., et al. 2005. Matricellular homologs in the foreign body response: hevin suppresses inflammation, but hevin and SPARC together diminish angiogenesis. Am. J. Pathol. 166:923-933.

61. Sage, E.H., et al. 2003. Cleavage of the matricellular protein SPARC by matrix metalloproteinase 3 produces polypeptides that influence angiogenesis. J. Biol. Chem. 278:37849-37857.

62. Norose, K., et al. 1998. SPARC deficiency leads to early-onset cataractogenesis. Invest. Ophthalmol. Vis. Sci. 39:2674-2680.

63. Sakurai, E., et al. 2003. Targeted disruption of the CD18 or ICAM-1 gene inhibits choroidal neovascularization. Invest. Ophthalmol. Vis. Sci. 44:2743-2749.

64. Sage, E.H. 2005. SPARC/osteonectin. In Current protocols in cell biology. K. Yamada, editor. John Wiley \& Sons Inc. Hillsborough, New Jersey, USA. In press. 10.11.1-10.11.23, Suppl. 17.

65. Sweetwyne, M.T., et al. 2004. Functional analysis of the matricellular protein SPARC with novel monoclonal antibodies. J. Histochem. Cytochem. 52:723-733.

66. Brekken, R.A., Huang, X., King, S.W., and Thorpe, P.E. 1998. Vascular endothelial growth factor as a marker of tumor endothelium. Cancer Res. 58:1952-1959. 